\title{
Síndromes de dolor uretral (SDU) y escrotal (SDE)
}

\author{
Ruiz Cerdá JL. \\ Servicio de Urología. Hospital Universitario La Fe. Valencia.
}

Actas Urol Esp. 2007;31(4):338-344

\section{RESUMEN}

SÍNDROMES DE DOLOR URETRAL (SDU) Y ESCROTAL (SDE)

El dolor pélvico no es una entidad infrecuente. El dolor uretral y el escrotal forman parte, junto a la prostatitis y el síndrome de vejiga dolorosa o cistitis intersticial, al conjunto de síndromes de dolor pélvico. El objetivo de esta revisión es realizar una puesta al día de los conocimientos actuales disponibles sobre epidemiología, etiología, diagnóstico y tratamiento para el síndrome uretral y escrotal. Los datos epidemiológicos para ambos síndromes son muy escasos. Carecemos de una definición uniforme o de un marcador diagnóstico de enfermedad y la etiología permanece sin esclarecer. Aunque, podría tratarse de un dolor neuropático sin un claro estímulo específico o un dolor desproporcionado en intensidad al estímulo. En cuanto al diagnóstico, no hay evidencia que permita clasificar adecuadamente a los pacientes o cuantificar los síntomas para incluir o excluirlo a un determinado enfermo. No obstante, es muy importante conocer la neuroanatomía funcional para la evaluación de estos pacientes. El tratamiento debe ser siempre primariamente conservador. La cirugía se debe evitar siempre que sea posible. Como no existe un tratamiento específico, la disminución del dolor es el principal objetivo, por lo que el urólogo debe conocer el manejo de los analgésicos mayores, los antidepresivos y los anticonvulsivantes.

Palabras clave: Dolor uretral. Dolor escrotal.

\section{ABSTRACT \\ URETHRAL AND SCROTAL PAIN SYNDROMES}

Painful diseases of the pelvis are not rare. Urethral and scrotal pains are part of the chronic pelvic pain syndromes along with chronic prostatitis and painful bladder syndrome/interstitial cystitis. The objective of this review is to update the current knowledge about epidimiology, etiology, diagnosis and best treatment for patients with urethral and scrotal pain syndromes. Epidemiological data for both syndromes are scant. We lack of uniform definition or readily available diagnostic marker and aetiology remains unclear. The pain could be a neuropathic without a specific stimulus or disproportionate to the stimulus intensity. Regarding diagnosis, there is no evidence to qualify or quantify the symptoms to include or exclude patients. Nevertheless, it is very important to know functional neuroanatomy in the evaluation of these patients. Treatment always should be primary conservatively. Surgery should be avoided if possible. As no specific treatment is available, decrease the pain is the main objective, therefore urologist should know how to manage analgesics, antidepressant and anticonvulsants. 


\section{SÍNDROME DE DOLOR URETRAL (SDU)}

El Subcomité de Estandarización de la Sociedad Internacional de Continencia definió el síndrome de dolor uretral (SDU), en terminología de tracto urinario inferior, como el episodio persistente o recurrente de dolor uretral regular normalmente al miccionar, con aumento de frecuencia diurna y nocturia, en ausencia de infección $u$ otras patologías conocidas ${ }^{1}$. Se puede encuadrar como parte de los síndromes de dolor pélvico crónico. Los datos epidemiológicos son escasos. Carecemos de definición uniforme, marcador diagnóstico fácilmente disponible, etiología conocida, fisiopatología probable, metodología estandarizada y uniformidad en el tamaño de la población objeto de estudio. Es principalmente un sindrome que afecta a mujeres en edad fértil como una media de edad de 39 años $^{2}$.

\section{Etiologia}

La etiología de SDU es confusa a pesar de que se han propuesto muchas teorías con poco respaldo científico. Una tercera parte de los pacientes tienen antecedentes familiares de problemas de polaquiuria ${ }^{2}$. La teoría de que los síntomas son causados por obstrucción uretral y son quirúrgicamente tratables ha tenido muchos defensores $^{3}$. Sin embargo, no existe evidencia que apoye una obstrucción anatómica y los estudios que informan de unos resultados excelentes después de una dilatación uretral, carecen de uniformidad en el criterio diagnóstico de obstrucción y están muy poco documentados. Aunque, ocasionalmente se ha informado de casos con buena respuesta a alfa-bloqueantes ${ }^{4}$.

El papel de la infección ha sido investigado por muchos autores, pero existe poca evidencia que apoye el concepto de síndrome de etiología inflamatoria o infecciosa ${ }^{5}$. No obstante, se ha descrito mejoría en los síntomas después de terapia antibiótica en un alto porcentaje de pacientes ${ }^{6}$. La identificación de un microorganismo es crucial para sustentar esta teoría. Solamente se ha identificado un organismo patógeno en un 15\% de los pacientes. Aunque en casos de origen bacteriano se puede demostrar una sobreinfección ${ }^{7}$. Un dato indirecto que apoya la infección como responsable del SDU, es el hallazgo de leucoplasia trigo- nal. Aunque la literatura en relación a este hallazgo cistoscópico es escasa y controvertida, se ha asociado con infección crónica.

Otra posible etiología es la que considera el SDU como una manifestación de una forma menos grave o de una fase precoz de cistitis intersticial $(\mathrm{CI})^{8}$. Esta teoría se basa en la idea de que cualquier paciente, hombre o mujer con sintomatología de urgencia miccional y/o dolor pélvico, sin importar donde se percibe si en la vejiga o en la uretra, sufren fundamentalmente de un desorden fisiopatológico llamado "Disfunción Epitelial del Tracto Urinario Inferior". Esta disfunción epitelial permite que los solutos de la orina se filtran en los espacios subepitelial y muscular. A pesar de que los datos en los que el factor urinario es el responsable de los síntomas no son concluyentes, el potasio podría ser el origen del daño tisular. No solo en el epitelio de la vejiga sino también en los epitelios prostático y uretral donde el potasio estimula las terminaciones nerviosas del dolor. Por tanto, un mismo proceso patológico podría ser el responsable de la CI, SDU y de la prostatitis. Los datos que respaldan esta teoría son la similitud de sintomas, tests de potasio positivos similares y la relación entre la gravedad de los sintomas y los test de provocación con potasio en la CI comparándolo con el SDU. Además, según se ha informado, la uretra es la localización básica del dolor en un $74 \%$ de los pacientes de $\mathrm{CI}^{9}$.

Al igual que en otros síndromes de dolorosos con ausencia de patología orgánica conocida, se han propuesto teorías relacionadas con un origen psicogénico. El papel de los factores psicológicos se ha estudiado en pacientes con dolor genital crónico, revelando altos niveles de hipocondría, depresión-somatización y falta de apoyo social. En la actualidad, existe evidencia, con base bioquímica, respecto a vías serotonérgicas que podrían jugar un papel en los síndromes de dolor pélvico crónico. Además, sustenta esta teoría la buena respuesta del dolor neuropático a la amitriptilina o duloxetina.

\section{Diagnóstico}

El diagnóstico de SDU es clínico y basado en la sintomatología así como un diagnóstico por exclusión. Después de la evaluación médica, los 
especialistas no suelen descubrir hallazgos específicos que justifiquen o expliquen los sintomas. No hay evidencia para calificar o cuantificar los síntomas para incluir o excluir pacientes del diagnóstico de SDU. Las mujeres son frecuentemente jóvenes y activas sexualmente. Los síntomas pueden ser intermitentes y caracterizados por urgencia y frecuencia, dolor uretral persistente y/o pélvico. Se ha informado de dolor durante el coito en el 50\% de los pacientes ${ }^{2}$.

La labor a realizar se basa en descartar cualquier otra enfermedad orgánica, funcional, inflamatoria, tumoral o infecciosa responsable de los síntomas. Es necesaria una historia detallada respecto a las características y temporalidad del dolor, patrón de frecuencia, intensidad, duración desde el comienzo, cualquier posible etiologia que pueda incluir alguna causa que precipite o que provoque el dolor. El examen ginecológico junto con una exploración del suelo pélvico en posición de litotomía debería incluir un examen rectal. Se recomienda asimismo el examen neurológico de los nervios que llegan a la región genital para identificar déficit de inervación en el área urogenital $^{10}$. Es necesario realizar análisis de orina, cultivos para micobacterias, exudados uretrales, vaginales y de cérvix y análisis séricos para Clamidia, Ureaplasma, Micoplasma, Virus humanos del papiloma y herpes, citología urinaria y análisis de orina microbiológicos tras lavado vesical. Alrededor del 30\% de los casos pueden tener leucocituria y el $25 \%$ microhematuria ${ }^{6}$. El concepto aceptado de bacteriuria significativa corresponde a más de $10^{5}$ unidades formadoras de colonias (UFC) $/ \mathrm{ml}$. Sin embargo, podría ser suficiente con $10^{2}$ de una única especie de un patógeno conocido.

Están indicados estudios radiológicos o ecográficos para descartar causas secundarias. El estudio urodinámico con electromiografía perineal es necesario para evaluar funcionalmente el tracto urinario inferior y descartar residuo miccional importante o poca relajación del esfinter uretral $^{11}$. No se recomienda el uso de Test de sensibilidad intravesical al potasio como herramienta diagnóstica en el SDU. El test de sensibilidad intravesical al potasio solo indica el aumento de permeabilidad y únicamente el 55\% de los pacientes tienen un test positivo. Sin embargo, puede ayudar a indicar tratamiento con heparina como primera opción para corregir la disfunción epitelial. La cistoscopia revela a menudo hiperemia y aumento de la vascularización uretral, seudo-papilomas en el cuello de la vejiga y metaplasia escamosa trigonal y leucoplaquia ${ }^{6}$. La evaluación psicológica debería formar parte de la evaluación multidisciplinaria para detectar síntomas de depresión asociados con el SDU.

\section{Tratamiento}

Las estrategias de tratamiento del SDU incluyen abordajes conservadores y quirúrgicos. Se recomienda la terapia sistémica con anticolinérgicos, alfa-adrenérgicos y relajantes musculares. Aunque, cuando los pacientes llegan al urólogo todos ellos suelen haber fracasado. La terapia antibiótica oral mas un bactericida vaginal y/o agente antimitótico se ha propuesto como tratamiento en caso de leucoplaquia trigonal. Se basa en la hipótesis de que la leucoplaquia es un signo de infección crónica subyacente. La terapia de larga duración con $100 \mathrm{mg}$ de doxiciclina dos veces al día durante dos semanas, seguida de $100 \mathrm{mg}$ una vez al día durante otras dos semanas ha reportado un $70 \%$ de curación o mejoría ${ }^{6}$. La doxiciclina es un antibiótico de amplio espectro que es efectivo contra los microorganismos que no son detectados en los cultivos de orina rutinarios (C.Trachomatis, U. Urealyticum, M. Genitalium).

La terapia heparinoide con heparina intravesical, polisulfato de pentosan (PPS) intravesical o PPS oral podría estar indicada cuando el dolor uretral está en el contexto de una CI. Se recomienda terapia de larga duración durante meses o incluso 1-2 años ${ }^{2}$. La inclusión de un antihistamínico (hidroxizina) puede beneficiar a un 65\% de pacientes cuando existe disfunción epitelial ${ }^{8}$.

La terapia física ha sido evaluada en pacientes con CI y SDU. El razonamiento se basó en la hipótesis de que los puntos miofasciales detonanes en el suelo pélvico no son sólo una fuente de dolor y de sintomas urinarios, sino que también provocan inflamación neurogénica de la vejiga a través de reflejos o impulsos antidrómicos. La terapia manual del suelo pélvico para la disminuir la hipertonía del suelo pélvico puede mejorar 
los sintomas de SDU en aproximadamente un $80 \%$ de los pacientes ${ }^{12}$. Si están disponibles, también puede ayudar a algunos pacientes la acupuntura manual o electroacupuntura ${ }^{13}$. Además se han propuesto en casos de espasmo del esfinter externo, electroestimulación y biofeedback. Sin embargo, no existe buena evidencia de sus resultados ${ }^{14}$.

En los casos de trigonitis, la diatermocoagulación endoscópica y la crioterapia pueden ser beneficiosas para tratar de restablecer un urotelio normal. El láser Nd-YAG es también otra alternativa que ha demostrado la regeneración del epitelio funcional normal y mejoría en los síntomas en un $78 \%$ de los pacientes ${ }^{15}$. Es importante recordar que se puede producir una remisión espontánea.

Se cree que los síndromes de dolor crónico pueden, en última instancia, resultar en sensibilización central y convertirse en crónicos y sin relación con el órgano que originó la primera patología. Cuando fallan los opioides y anti-inflamatorios no-esteroides (AINES) pueden ayudar nuevos tratamientos analgésicos efectivos como carbamazepina y gabapentina, o antidepresivos como la amitriptilina y la duloxetina.

\section{SÍNDROME DE DOLOR ESCROTAL (SDE)}

El Subcomité de Estandarización de la Sociedad Internacional de Continencia definió el síndrome de dolor escrotal (SDE), en terminología de tracto urinario inferior, como el episodio de dolor persistente o recurrente y regular asociado con síntomas relacionados con el tracto urinario o con disfunción sexual. No debe haber orquiepididimitis demostrada $\mathrm{u}$ otra patología conocida ${ }^{1}$.

El dolor tiene debe tener una duración de al menos 6 meses para calificarlo como dolor escrotal crónico, aunque una duración de 3 podría ser suficiente ${ }^{16}$. Debe interferir con las actividades diarias y con la calidad de vida del paciente. La única información disponible se refiere a la prevalencia del síndrome postvasectomía. En estudios retrospectivos, utilizando cuestionarios enviados por correo puede llegar a representar hasta un 15-19\% de los pacientes ${ }^{17,18}$.

\section{Etiologia}

El dolor escrotal crónico puede ser debido a causas testiculares concretas, dolor referido o idiopático. Las causas posibles de dolor testicular y/o referido son amplias (Tabla 1). Los testículos comparten su inervación con la cabeza y el cuerpo del epidídimo recibiendo sus terminaciones nerviosas del plexo espermático superior, vía fibras nerviosas, que acompañan a los vasos espermáticos internos con aportes del plexo espermático inferior, plexo hipogástrico superior y otros ganglios de la cadena simpática pélvica. La lámina parietal y visceral de la túnica vaginalis y el cremaster reciben inervación aferente originada en L1-L2, conducida por la rama genital del nervio genito femoral. Este nervio tiene de una rama femoral que inerva la piel de la región ventromedial de la cadera y una rama genital a la región escrotal. El nervio ilioinguinal transporta la sensación desde la región de la ingle. El nervio pudendo inerva el área cutánea perineal. Cualquier órgano que comparte la misma inervación que el contenido escrotal puede provocar

Tabla 1

Diagnósticos diferenciales del síndrome de dolor escrotal

Lesiones intra-escrotales palpables

- Tumor Testicular

- Hidrocele

- Espermatocele

- Quiste de epidídimo, en la túnica albugínea o en el cordón espermático

- Varicocele

Lesiones evidentes en la ecografía

- Áreas hipo-hiperecoicas, No homogeneidad

- Microlitiasis testicular

Cirugía previa

- Reparación de hernia

- Vasectomía (Síndrome de dolor post-vasectomía)

Lesiones extragenitales

- Enfermedad de la columna

- Litiasis ureteral

- Aneurisma aórtico o ilíaco

- Estreñimiento

Hipermovilidad de los testículos

- Subtorsion

Causas neurogénicas

- Atrapamiento del nervio pudendo

Dolor pélvico crónico de causa desconocida

- Dolor escrotal

- Dolor testicular

- Dolor epididimario 
dolor en esta región. Desafortunadamente, en una gran proporción de pacientes la etiología del dolor escrotal es desconocida y así nos enfrentamos al SDE.

El SDE es fácilmente reconocible pero su fisiopatología se entiende poco. Se considera parte de un más complejo dolor neuropático urogenital. El papel de la inflamación neurogénica ha sido implicado en su génesis. La inflamación neurogénica se provoca mediante la activación de las fibras sensoriales amielínicas a través de estímulos nocivos que liberan neuropéptidos. Como continuación a la lesión nerviosa ocurren cambios neurofisiológicos en el sistema nervioso autónomo que pueden persistir indefinidamente. Bajo estas condiciones el dolor puede suceder sin estímulos específicos o puede ser desproporcionado a la intensidad del estímulo.

Por otra parte, se ha sugerido la hipótesis de que cambios en las propiedades de los receptores alfa2-presinápticos pueden estar implicados en el dolor testicular ${ }^{19}$. El conducto deferente humano obtenido de pacientes con SDE, mostraban sensibilidad reducida a la rauwosclina, un alfa2antagonista selectivo. Aunque se reconoce que los síntomas psicológicos pueden estar implicados en la génesis del dolor testicular, no hay evidencia de la importancia relativa de los parámetros psicológicos en el desarrollo y mantenimiento del dolor testicular crónico ${ }^{10,16}$.

\section{Diagnóstico}

Se recomienda una evaluación diagnóstica minuciosa dirigida por la historia clínica y el examen físico. Además de los estudios urológicos, el trabajo diagnóstico debería incluir exploraciones gastro-intestinales para descartar el dolor referido de órganos pélvicos inferiores y palpación de hernia. Asimismo esta indicada una exploración neurológica para descartar un problema de dolor neuropático involucrando las raíces lumbosacras, nervio ilioinguinal, genito femoral, pélvico o pudendo. La tomografía computarizada (TC) y la resonancia nuclear magnética (RNM) pueden ayudar para valorar una posible hernia discal o la sospecha de un aneurisma como causeas del problema de dolor crónico. La evaluación multidisciplinar debería incluir valoración psicológica o psiquiátrica para descartar depresión. El SDE afecta a muchos aspectos de la vida del hombre y es crucial que, si están presentes, los síntomas depresivos iniciales se traten apropiadamente. A pesar de la realización de estudios minuciosos no se ha hallado la etiología del dolor en el SDE.

$\mathrm{El}$ dolor escrotal puede ser unilateral o bilateral, continuo o intermitente. Para el especialista que tiene que valorar un paciente con SDE es importante comprender la inervación testicular, para que el diagnóstico pueda guiarse por la neuroanatomía funcional. Se debe realizar una palpación suave para identificar cada componente del escroto. No es infrecuente localizar el punto y distinguir entre dolor epipidimario y testicular. Es obligatorio realizar un tacto rectal y se debe examinar la integridad de la pelvis. Los análisis de laboratorio estándar deben incluir lo siguiente: análisis de orina con cultivo, PSA en varones mayores de 50 años, exudado uretral o PCR para gonorrea y clamidia.

La ecografia escrotal debe ser realizada como estudio base para buscar lesiones o cambios testiculares o epididimarios. Sin embargo, en ausencia de hallazgos significativos clínicos durante el examen físico y en presencia de análisis de orina negativos, el único beneficio real del ultrasonido escrotal es la tranquilizar al paciente preocupado por el posible etiología tumoral ${ }^{21,22}$.

\section{Tratamiento}

El SDE debe tratarse en principio de forma conservadora. Se deberá evitar en lo posible la cirugía. Aunque no se ha identificado infección, un pequeño número de pacientes puede responder a una combinación de fármacos antibióticos y AINES. Los antidepresivos tricíclicos algunas veces alivian el dolor. Aquellos con síntomas intratables pueden beneficiarse del enfoque de un equipo multidisciplinar que implique a un urólogo, un especialista clínico del dolor y un psicólogo. El uso de gabapentina o carbamazepina necesita la consulta de un especialista del dolor. Se ha probado la analgesia TENS con buenos resultados $^{23}$. Se pueden probar en régimen ambulatorio. Un bloqueo del cordón espermático con una mezcla de lidocaína al $1 \%$ sin adrenalina y $1 \mathrm{ml}$ de metilprednisolona. El procedimiento, si tiene éxito, se puede repetir a intervalos regulares. 
Se ha defendido que el SDE en algunos pacientes podría ser el resultado de un espasmo/obstrucción funcional a lo largo del conducto deferente y del epidídimo. Se ha demostrado que el conducto deferente del escroto humano tiene una distribución similar de receptores que el músculo liso prostático ${ }^{24}$. Por consiguiente, los fármacos que modifican la actividad de las fibras musculares lisas prostáticas deberían también afectar al deferente. Para los pacientes que no responden al manejo conservador, un tratamiento prueba con un alfa-bloqueante podría ser una opción antes de plantear un tratamiento quirúrgico.

Las opciones de tratamiento médico dan buenos resultados habitualmente y solo unos pocos pacientes necesitan procedimientos quirúrgicos como último recurso. La denervación del cordón espermático implica la división del nervio ilioinguinal y de sus ramas. Incluso en el caso de una vasectomía previa, para erradicar la inervación simpática que puede contribuir a una distrofia simpático refleja. También se puede realizar una denervación laparoscópica. Los vasos gonadales se aíslan circunferencialmente y se dividen próximamente al conducto deferente y a su vascularización. Se puede alcanzar una reducción del dolor en aproximadamente el 70\% de los $\operatorname{casos}^{25,26}$. La epididimectomía se debe realizar sólo en el caso que el paciente haya sido advertido de controvertidos resultados publicados ${ }^{27,28}$. Hay pacientes responden a la epididimectomía. Se cree que la presencia de cambios inflamatorios crónicos puede ser precursora de malos resultados. La vasovasostomía podría ser una opción en casos refractarios de orquialgia postvasectomía ${ }^{29}$. Desafortunadamente un pequeño número de pacientes que no responden a tratamiento medico o más invasivo tendrán que ser sometidos, en última instancia, a orquiectomía inguinal, para aliviar el dolor. Este procedimiento debe ser el último recurso y claramente compromete la situación si el paciente desarrolla posteriormente síntomas contralaterales.

\section{REFERENCIAS}

1. Abrams P, Cardozo L, Fall M, Griffiths D, Rosier P, Ulmsten U, van Kerrebroeck P, Victor A, Wein A. The standardisation of terminology of lower urinary tract function. The International Continence Society Committee on Standardisation of Terminology. Am J Obstet Gynecol 2002;187:116-126.
2. Parsons CL, Zupkas P, Parsons JK. Intravesical potassium sensitivity in patients with interstitial cystitis and urethral syndrome. Urology 2001; 57(3):428-433.

3. Sand PK, Bowen LW, Osterganrd DR, Bent A, Panganibaun R. Cryosurgery versus dilation and massage for treatment of recurrent urethral syndrome. J. Reprod Med 1989; 34(8):499-504.

4. Sinha D, Arunkalaivanan A. Urethral syndrome: response to alpha-adrenergic blocking agents. Int Urogynecol J Pelvic Floor Dysfunct. 2006; 17(6):659-60.

5. Messinger EM. Urethral syndrome. In: PC Walsh, AB Retik, TA Stamey and ED Vaughan (Eds.), Campbell's Urology, W.B. Saunders, Philadelphia, 1992, pp. 997-1005.

6. Burkhard FC, Blick N, Hocreiter WW, Studer UE. Urinary urgency and frequency, and chronic urethral and/or pelvic pain in females. Can doxycicline help? J Urol 2004; 172(1):232-235.

7. Mutlu B, Mutlu N, Yucesoy G. The incidence of Chlamydia trachomatis in women with urethral syndrome. Int $\mathrm{J}$ Clin Pract 2001; 55(8):525-6.

8. Parsons CL. Prostatitis, interstitial cystitis, chronic pelvis pain and urethral sindrome share a common pathophysiology: lower urinary dysfunctional epithelium and potassim recycling. Urology 2003; 62(6):976-982.

9. Fitzgerald MP, Brensinger C, Brubaker L, Propert K, ICDB study group. What is the pain of interstitial cystitis like? Int Urogynecol J Pelvic Floor Dysfunct 2006; 17(1):69-72.

10. Wesselmann U, Burnett AL, Heinberg. The urogenital and rectal pain syndromes. Pain 1997; 73(3):269-294.

11. Kuo HC. Urodynamic study and potassium sensitivity test for women with frequency-urgency syndrome and interstitial cystitis. Urol Int, 2003, 71(1):61-5.

12. Weis JM. Pelvic floor myofascial trigger points: manual therapy for interstitial cystitis and the urgency-frequency syndrome. J Urol 2001, 166(6):2226-31.

13. Chen YL, Ha LF, Cen J, Huang QR, Hou WG, Gao ZQ. Comparative observation on therapeutic effects of electroacupuncture and manual acupuncture on female urethral syndrome. Zhongguo Zhen Jiu. 2005; 25(6)425-426.

14. Kaplan WE, Firlit CF, Schoenberg HW. The female urethral syndrome: external sphincter spasms as etiology. J Urol 1980; 124:48-49.

15. Constantini E, Zucchi A, Del Zingaro M, Mearini L. Treatment of urethral syndrome: a prospective randomized study with ND:YAG laser. Urol Int 2006; 76(2):134-8.

16. Granitsiotis P, Kirk D. Chronic testicular pain: an overview. Eur Urol 2004; 45(4):430-436.

17. Gingell C, Crosby D, Carroll R. Review of the complications and medicolegal implications of vasectomy. Postgrad Med J 2001; 77(912):656-659.

18. Manikandan R, Srirangam SJ, Pearson E, Collins GN. Early and late morbidity after vasectomy: a comparison of chronic scrotal pain at 1 and 10 years. BJU Int 2004; 93(4):571-574.

19. Granitsiotis P. Sensitivity of the pre-junctional alfa2-adrenoceptors: is it different in patients with testicular pain? Poster presented at the Urological Research Society. 2001.

20. Fall M, Baranowski AP, Fowler CJ, Lepinard V, Malone-Lee JG, Messelink EJ, Oberpenning F, Osborner JL, Schumacher S. Guidelines on Chronic Pelvic Pain. In: European Association of Urology Guidelines, 2006 Edition, p.62-124. 
21. Van Haasrt EP, van Andel G, Rijcken TH, Schlatmann TJ, Taconis WK. Value of diagnostic ultrasound in patients with chronic scrotal pain and normal findings on clinical examinatiom. Urology 1999; 54(6):1068-1072.

22. Lau MWM, Taylor M, Payne SR. The indications for scrotal ultrasound. Br J Radiol 1999; 72(861):833-8377.

23. Macrae B. Pain: new thoughts on an old problem. Urology News, 2000;4:6-8.

24. Granitsiotis P, Daly C, Kirk D, McGrath JC. Characterization of postjunctional receptors along the length of the human vas deferens: differences from the roedents. BJU Int 2000; 83:365.

25. Levine LA, Matkov TG. Microsurgical denervation of the spermatie cord as primary surgical treatment of chronic orchialgia. J Urol, 2001 165(6pt1):1927-1929.

26. Chadeddu JA, Bishoff JT, Chan DY, Moore RG, Kavoussi LR, Jarret TW. J Urol 1999; 162 (3pt1):733-735.
27. Padmore DE, Norman RW, Miliard OH. Analyses of indications for and outcomes of epididymectomy. J Urol 1996; 156(1):95-96.

28. West AF, Leung HY, Powell PH. Epididymectomy is an effective treatment for scrotal pain after vasectomy. BJU Int 2000; 85(9): 1097-1099.

29. Davis BE, Noble MJ, Weigel JW, Foret JD, Mebust WK. Analysis and management of chronic testicular pain. $\mathrm{J}$ Urol 1990; 143(5):936-9.

Correspondencia autor: Dr. J.L. Ruiz Cerdá

Hospital Universitario La Fe

Avda. Campanar, 21. 46009 Valencia

Tel.: 963862700

E-mail autor: Jose.L.Ruiz@uv.es

Información artículo: Revisión

Trabajo recibido: febrero 2007

Trabajo aceptado: marzo 2007 